\section{Case Report}

Journal of Epilepsy Research pISSN 2233-6249 / elSSN 2233-6257

Received September 10, 2019

Revised October 21, 2019

Accepted January 9, 2020

Corresponding author:

Kanij Fatema, FCPS

Department of Pediatric Neurology,

Bangabandhu Sheikh Mujib Medical

University, House 37, Road 10A,

Dhanmondi, Dhaka 1209, Bangladesh

Tel. +8801713097751

Fax. +8801713097751

E-mail; mailmonami@gmail.com

\title{
SCN8A Mutation in Infantile Epileptic Encephalopathy: Report of Two Cases
}

\author{
Kanij Fatema, FCPS, Md Mizanur Rahman, FCPS, Omar Faruk, MBBS \\ Department of Pediatric Neurology, Bangabandhu Sheikh Mujib Medical University, Dhaka, Bangladesh
}

\begin{abstract}
Early infantile epileptic encephalopathy type 13 is a severe form of epilepsy caused by mutations in the sodium channel 8 alpha (SCN8A) gene. This gene encodes the neuronal voltage-gated sodium channel which plays vital role in neuronal excitability. Here we present two cases with SCN8A encephalopathy. Both cases had mutation in p.Arg1872Gin the SCN8A gene, which was detected by targeted next generation sequencing. Case 1 was a 14-month old boy, who had a normal birth history with normal development up to 6 months and then developed repeated generalized seizure, which was nonresponsive to multiple antiepileptic drugs. He also had neuroregression and dystonia. His electroencephalogram (EEG) showed progressive background abnormality with burst suppression pattern. His metabolic panel was normal and had partial response to carbamazepine. The second case was for an 11-month old boy with the onset of seizure at the age of 7 months. Seizure was generalized, resistant to multiple antiepileptic drugs. He had developmental delay from beginning, no movement disorder. EEG showed focal discharge from left temporal and occipital region. He showed partial response to oxcarbazepine. Our cases had similarities with the previously reported cases. The detailed discussion of our cases would contribute to early detection and targeted treatment of SCN8A encephalopathy. This also gives special emphasis on a genetic test in infants with intractable epilepsy, movement disorder and developmental delay. (2019;9:147 -151)
\end{abstract}

Key words: Sodium voltage-gated channel alpha subunit 8 (SCN8A), Epileptic encephalopathy, Epilepsy

\section{Introduction}

Early infantile epileptic encephalopathy (EIEE) is a severe form of epilepsy, which affects children in early infancy and causes devastating neurological disorders. They have variable genetic heterogeneity. ${ }^{1,2}$ Sodium voltage-gated channel alpha subunit 8 (SCN8A) mutation causing EIEE type 13 comprises $1 \%$ of patients of epileptic encephalopathy. ${ }^{3}$ SCN8A-encephalopathies are autosomal dominant disorders, which is caused by de novogain-of-function missense mutations in the gene. This gene is part of the voltage gated sodium channels (VGSCS) gene family. ${ }^{4,5}$ VGSCs plays important role in controlling neuronal excitability. They are primarily expressed in the central nervous system and mutation causes epilepsy. ${ }^{6-9}$

SCN8A gene is located at human chromosome 12q13.13. The coding sequence (170 Kb) of this gene contains 27 exons which encodes a protein of 1980 amino acid residues, intolerant to variations. ${ }^{10-12}$ Thus a minor deviation in the properties of the ion channel proteins can lead to significant functional defects. ${ }^{13}$ SCN8Aencodes the Nav $1.6 \alpha$ subunit of VGSCs, which is highly expressed in the initial axonal segment and nodes of Ranvier of myelinated axons, and it is responsible for generation of action potential and conduction. ${ }^{14-16}$

The clinical presentation of SCN8A mutation is variable. The common features are intractable epilepsy, which is evident in infancy along with developmental stagnation or delay. However, some cases are milder, which presents as benign infantile seizures and paroxysmal choreoathetosis. While some cases present only with intellectual disability. ${ }^{14-16}$

In this research, we presented that the clinical and genetic features of two cases of SCN8A encephalopathy, presented with intractable epilepsy and developmental delay.

\section{Case Report}

\section{Case 1}

A 14 months old male child, only issue of his nonconsanguineous parents, partially immunized presented with recurrent attacks of seiz- 
ure since 6 months of age. Seizure was generalized tonic clonic in nature with upward rolling of eyes and persisted for about 1 to $2 \mathrm{mi}$ nutes, occurring four to five times per day. He was born with normal vaginal delivery at term, with average birth weight. There was no adverse event in the perinatal period. Before the onset of seizure, his development was normal for his age. Since then, there was progressive regression of achieved milestones and he lost his neck control, social smile and was least responsive to surroundings. Along with this, he also developed involuntary movement manifested by posturing mainly in limbs, which occurred during awake period and subsided in sleep.

There was no history excessive vomiting, abnormal urine or body odor, loss of consciousness, fever, feeding difficulty, respiratory tract infection, skin changes etc. On examination the child was conscious, there was no facial dysmorphism. His vitals were withinnormal limit and he was anthropometrically well thrived. Bacillus Calmette-Guérin (BCG) mark was present without any skin stigmata. Developmental assessment revealed that there was no neck control or social smile but the child could fix, follow and localize sound, but no babbling. On nervous system examination, there was hypertonia in all four limbs with exaggerated deep tendon reflexes and planter was extensor. He also had dystonia in both upper and lower limbs. Other systemic examinations revealed nothing abnormal and ophthalmological examination was also normal. Psychological examination using a third edition of the Bayley Scales of Infant and Toddler Development (BSID-III) revealed cognition was around 2 months, gross motor 3 months and fine motor 3 months level.

A complete blood count test showed hemoglobin level of $12 \mathrm{~g} / \mathrm{dL}$ with total leukocyte count of $10,000 / \mathrm{cm}^{2}$ and platelet of $450,000 / \mathrm{cm}^{2}$. Initial 2 electroencephalogram (EEG) showed focal discharges from frontal and temporal areas with apparently normal background. But in the last EEG, there was burst of epileptic discharges, arising from frontal and temporal regions with severe background abnormality evident by attenuation and slowing mostly in posterior regions. The brain magnetic resonance imaging (MRI) showed cortical atrophy. Serum ammonia was normal, serum lactate was normal, urinary ketone body was negative, random blood sugar (RBS) was normal, arterial blood gas (ABG) was normal. Tandem mass spectrometer (TMS) was within normal limits, biotinidase enzyme activity was within normal limits and gas chromatography mass spectrometry (GCMS) was within normal limits.

Genetic study with clinical exome sequencing revealed there was mutation on SCN8A(+) at exon 27, variant c.5615G>A (p.Arg1872Gin), zygosity heterozygous. Disease as per Online Mendelian Inheritance in
Man (OMIM) was EIEE type 13, inheritance-autosomal dominant, classification-likely pathogenic.

\section{Treatment}

He was treated with phenobarbitone, levetiracetam, prednisolone, adenocorticotropic hormone, vigabatrine and sodium valproate, but seizure was not controlled. Later he was treated with carbamazepine, which caused the $50 \%$ control of the seizure. For dystronia, trihexyphenydyl was given to the child and for developmental delay, physiotherapy was given.

\section{Case 2}

An 11 months old male child, who had the 3rd issue of his non consanguineous parents partially immunized presented with recurrent attacks of seizure since 7 months of age which was generalized tonic clonic in nature with upward rolling of eyeball, occurred 5-6 times/day, each episode persisted for about 1-2 minutes.

He had the delayed developmental milestone manifested by neck control, achieved at 8 months old, and is unable to sit until the present. His birth history was uneventful. He had a history of unexplained sib death in neonatal period and his mother had a history of epilepsy in childhood. There was no medical history of excessive vomiting, abnormal urine or body odor, unconsciousness, fever, feeding abnormality, respiratory tract infections, skin manifestation, trau$\mathrm{ma}$, altered sensorium etc.

On examinations the child was conscious, without any facial dysmorphism. His vitals were within normal and he was anthropometrically well thrived. BCG mark was present without any skin stigmata. Developmental assessment revealed global developmental delay. On nervous system examination, there was hypertonia with exaggerated deep tendon reflexes in all four limbs. Other systemic examinations revealed nothing abnormal. Ophthalmological examination was normal.

\section{Investigations}

CBC was normal, EEG showed focal epileptiform discharge arising from left temporal and occipital region, MRI of brain showed cortical atrophy, serum ammonia was normal, serum lactate, was normal, urinary ketone body was negative, RBS was normal, ABG was normal. TMS was within normal limits, Biotinidase enzyme activity was within normal limits. GCMS was normal.

Genetic study with clinical exome sequencing revealed SCN8A(+) mutation at exon 27, variant c.5615G>A (p.Arg1872Gin), zy- 
gositiy-heterozygous. Disease as per OMIM was EIEE type 13, inheritance-autosomal dominant, classification-likely pathogenic.

\section{Treatment}

He was treated with phenobarbitone, levetiracetam, vigabatrine, but there was minimal response. There was partial response the oxcarbazepine.

\section{Discussion}

SCN8A encephalopathy has been first described in 2012 and several cases have been identified till date. ${ }^{17}$ This encephalopathy is also known as EIEE type 13. The patients present with drug resistant seizure between age 3 and 7 months (mean age, 5 months; range, 1 day to 18 months). ${ }^{1,18,19}$ The seizure has no definite pattern but is often provoked. The common seizures are epileptic spasm, tonic, atonic, myoclonic, focal and absence seizure. ${ }^{1}$

The patients also present with developmental delay and/or stagnation. For the half of the patients, development is normal prior to the onset of seizure. In addition to that, there are movement disorder like ataxia, choreoathetosis, hypotonia, spasticity, dystonia and etc. ${ }^{1,12}$ Some patients present with behavioral disorder like autistic feature and etc. ${ }^{18,19}$ Whereas in literature some patients are found with milder phenotype such as early benign focal epilepsy, well controlled seizure, mild tremor, ataxia, motor delay and normal cognitive delay. $^{20}$

Clinically, two of our patients' phenotyepe are similar to the typical features of EIEE type 13. The age of onset is around 5 to 7 months. In one patient, the prior development was normal and in another, there was delay. In both of the patients, seizure was difficult to control and so multiple antiepileptic drugs were given. In one patient, movement disorder was present in the form of dystonia. In both of the infants, spasticity and global developmental delay were present. None of the patients had behavioral disorder like autism.

EEG findings are variable in EIEE type 13. Early EEG may be normal or show focal or multifocal epileptic discharges. EEG features change over time and the background shows progressive slowing. The epileptic discharges are most prominent in temporal regions, comprised of spike or spike followed by slow waves. There may be almost continuous slowing in temporal, parietal, and occipital regions. Superimposed beta waves and bilateral asynchronous spike or sharp waves may be found. ${ }^{1}$ There was progressive deterioration of the background in case 1, and also initially there was focal discharges, which turned into burst suppression pattern later on. In case 2, EEG showed focal epileptic discharges arising from occipital and temporal region; here the background was not uninterrupted.

Cranial MRI is not specific in SCN8A encephalopathy; usually it is normal in the most of the cases at the onset of seizure. However, there may be cortical atrophy or corpus callosum abnormality in some cases. There may be progressive cerebral or cerebellar atrophy in follow up imaging. ${ }^{1,21}$ In both of our cases cortical atrophy was found in MRI.

The final diagnosis is done by a genetic study. In targeted next generation sequencing, we identified heterozygous mutation in exon 27 of the SCN8A gene: c.5615G>A (p.Arg1872Gin) in cases 1 and 2. It represents a neocleotide substitution of guanine with adenine in chromosome 12, which results in amino acid substitution from arginine to glutamine at position 1872 of the protein, which is reported as a mutational hotspot. According to several groups of authors the amino acid substitution is severely damaging to the structure of the sodium channel. ${ }^{12}$

In previous literature it has been found that there is no obvious correlation between the positions of an SCN8A pathogenic variant and the seizure onset, seizure type or clinical severity. In one study significant similarity of phenotype was found in two unrelated individuals with same de novo pathogenic variant. ${ }^{21,22}$ However, in another study same pathogenic variant manifested different ages of the onset and disease severity. ${ }^{12}$

Penetrance of SCN8A encephalopathy which is identified until the present are de novo or inherited from either parent with a somatic mosaic variant, which is pathogenic and included in the germline. Also no pathogenic variant has been identified in an unaffected individual. ${ }^{23}$ The majority of the SCN8A mutation, which are found in genetic test, are gain-of-function causing the $\mathrm{Na}_{\mathrm{v}} 1.6$ channel to be hyperactive, and so there is excessive neuronal firing causing seizure. This mutation is also responsible for the intellectual disability as it has a primary neurodegenerative effect. ${ }^{24-26}$

Both our patients have been treated with multiple antiepileptic drugs before admission. Partial response has been noted in both cases with carbamazepine and oxcarbazepine. Literature has suggested that patients with SCN8A encephalopathy respond to the sodium channel blockers such as phenytoin, valproate, carbamazepine, lacosomide, lamotrigine, rufinamide and oxcarbazepine. ${ }^{1,27}$ In case 1, the patient did not respond to valproate but responded to carbamazepine partially. The case 2 did not respond to vigabatrin, phenobarbitone but responded partially to oxcarbazepine. In previous studies, 
most of the patients were maintained on multiple medications for partial seizure control. ${ }^{28}$ Boerma et al. ${ }^{28}$ reported four severely affected SCN8A encephalopathy patients who were successfully treated with high dose of phenytoin. Both phenytoin and carbamazepine is sodium channel blocker but the affinity of carbamazepine for sodium channel is about three times lower than phenytoin. ${ }^{29}$ We did not use phenytoin for any of our cases due to its side effects.

SCN8A encephalopathy has some consistent clinical and electrographic findings. Thus, in any case of intractable epilepsy in infancy with movement disorder and neuroregression or intellectual disability, where there is no definite etiology, a genetic test is suggested. This will be helpful in the precision medicine of the case as well as there will be a scope for genetic counseling for further pregnancy.

\section{References}

1. Larsen J, Carvill GL, Gardella E, et al. The phenotypic spectrum of SCN8A encephalopathy. Neurology 2015;84:480-9.

2. Wagnon JL, Barker BS, Hounshell JA, et al. Pathogenic mechanism of recurrent mutations of SCN8A in epileptic encephalopathy. Ann Clin Trans/ Neurol 2015;3:114-23.

3. Meisler MH, Helman G, Hammer MF, et al. SCN8A encephalopathy: research progress and prospects. Epilepsia 2016;57:1027-35.

4. Mantegazza M, Rusconi R, Cestele S. Mutations of ion channels in genetic epilepsies. In: Striano P, ed. Epilepsy towards the next decade: new trends and hopes in epileptology. Basel: Springer, 2015;15-34.

5. de Lera Ruiz M, Kraus RL. Voltage-gated sodium channels: structure, function, pharmacology, and clinical indications. J Med Chem 2015;58: 7093-118.

6. Scheffer IE, Zhang $\mathrm{YH}$, Jansen $\mathrm{FE}$, Dibbens L. Dravet syndrome or genetic (generalized) epilepsy with febrile seizures plus? Brain Dev 2009;31: 394-400.

7. Shi $X$, Yasumoto $S$, Kurahashi $H$, et al. Clinical spectrum of SCN2A mutations. Brain Dev 2012;34:541-5.

8. Nakamura K, Kato M, Osaka H, et al. Clinical spectrum of SCN2A mutations expanding to Ohtahara syndrome. Neurology 2013;81:992-8.

9. Vanoye CG, Gurnett CA, Holland KD, George AJ Jr, Kearney JA. Novel SCN3A variants associated with focal epilepsy in children. Neurobiol Dis 2014;62:313-22.

10. Plummer NW, Galt J, Jones JM, et al. Exon organization, coding sequence, physical mapping, and polymorphic intragenic markers for the human neuronal sodium channel gene SCN8A. Genomics 1998;54: 287-96.

11. O'Brien JE, Meisler MH. Sodium channel SCN8A (Nav1.6): properties and de novo mutations in epileptic encephalopathy and intellectual disability. Front Genet 2013;4:213.
12. Wagnon JL, Meisler MH. Recurrent and non-recurrent mutations of SCN8A in epileptic encephalopathy. Front Neurol 2015;6:104.

13. Meisler MH, Kearney JA. Sodium channel mutations in epilepsy and other neurological disorders. J Clin Invest 2005;115:2010-7.

14. Tzoumaka E, Tischler AC, Sangameswaran L, Eglen RM, Hunter JC, Novakovic SD. Differential distribution of the tetrodotoxin-sensitive $\mathrm{Rpn} 4 / \mathrm{NaCh6/Scn8a}$ sodium channel in the nervous system. J Neurosci Res 2000;60:37-44.

15. Gardella E, Becker F, Møller RS, et al. Benign infantile seizures and paroxysmal dyskinesia caused by an SCN8A mutation. Ann Neurol 2016; 79:428-36.

16. Trudeau MM, Dalton JC, Day JW, Ranum LP, Meisler MH. Heterozygosity for a protein truncation mutation of sodium channel SCN8A in a patient with cerebellar atrophy, ataxia, and mental retardation. J Med Genet 2006:43:527-30.

17. Atanasoska M, Vazharova R, Ivanov I, et al. SCN8A p. Arg1872Gln mutation in early infantile epileptic encephalopathy type 13: review and case report. Biotechnol Biotechnol Equip 2018;32:1345-51.

18. Arafat $A$, Jing $P, M a Y$, et al. Unexplained early infantile epileptic encephalopathy in Han Chinese children: next-generation sequencing and phenotype enriching. Sci Rep 2017;7:46227.

19. Horvath GA, Demos M, Shyr C, et al. Secondary neurotransmitter deficiencies in epilepsy caused by voltage-gated sodium channelopathies: a potential treatment target? Mol Genet Metab 2016;117:42-8.

20. Bagnasco I, Dassi P, Blé R, Vigliano P. A relatively mild phenotype associated with mutation of SCN8A. Seizure 2018;56:47-9.

21. Singh $R$, Jayapal $S$, Goyal $S$, Jungbluth $H$, Lascelles K. Early-onset movement disorder and epileptic encephalopathy due to de novo dominant SCN8A mutation. Seizure 2015;26:69-71.

22. Vaher $U$, Nõukas $M$, Nikopensius $T$, et al. De novo SCN8A mutation identified by whole-exome sequencing in a boy with neonatal epileptic encephalopathy, multiple congenital anomalies, and movement disorders. J Child Neurol 2014;29:NP202-6.

23. Hammer MF, Wagnon JL, Mefford HC, Meisler MH. SCN\&A-related epilepsy with encephalopathy. In: Adam MP, Ardinger HH, Pagon RA, et al. eds. GeneReviews $\Theta$. Seattle: University of Washington, 2020.

24. Blanchard MG, Willemsen MH, Walker JB, et al. De novo gain-of function and loss-of-function mutations of SCN8A in patients with intellectual disabilities and epilepsy. J Med Genet 2015;52:330-7.

25. Estacion $\mathrm{M}, \mathrm{O}$ 'Brien JE, Conravey $\mathrm{A}$, et al. A novel de novo mutation of SCN8A (Nav1.6) with enhanced channel activation in a child with epileptic encephalopathy. Neurobiol Dis 2014;69:117-23.

26. Takahashi S, Yamamoto S, Okayama A, et al. Electroclinical features of epileptic encephalopathy caused by SCN8A mutation. Pediatr Int 2015; 57:758-62.

27. Kong W, Zhang $Y$, Jiang $Y$. In response: SCN8A mutations in Chinese children with early onset epilepsy and intellectual disability. Epilepsia 2015;56:1320.

28. Boerma RS, Braun KP, van de Broek MP, et al. Remarkable phenytoin sensitivity in 4 children with SCN8A-related epilepsy: a molecular neuro- 
Fatema K, et al. SCN8A, Epileptic Encephalopathy 151

pharmacological approach. Neurotherapeutics 2016;13:192-7.

29. Kuo CC, Chen RS, Lu L Chen RC. Carbamazepine inhibition of neuronal
$\mathrm{Na}+$ currents: quantitative distinction from phenytoin and possible therapeutic implications. Mol Pharmacol 1997;51:1077-83. 